\title{
Comparaison de l'évolution morphologique entre les littoraux de : Béjaïa (Nord Est Algérien) et Saïdia (Nord Est Marocain)
}

\section{Cherif AOUDJ ${ }^{1,2}$, Fouzia HOUMA BACHARI ${ }^{1,2}$, Khadija AIT LEMQEDDEM ${ }^{3}$, Brahim DAMNATI ${ }^{3}$, Abla HANTAL ${ }^{1}$, Nour EI Islam BACHARI ${ }^{4}$ Abdelkarim MEZHOUD ${ }^{1,2}$, Rabah BELKESSA ${ }^{1,2}$}

1. Ecole Nationale Supérieure des Sciences de la Mer et de l'Aménagement du Littoral, ENSSMAL, Bois des Cars, B.P. 19 Dely Ibrahim, 16320, Algérie. cherifaoudj@gmail.com

2. Laboratoire de l'Écosystème Marin et Littoral, EcoSys, Chemin du complexe de Sidi Fredj, Staoueli 16096, Algérie.

3. Université Abdelmalek Essaadi, Faculté des Sciences et Techniques de Tanger, Département des Sciences de la Terre, Équipe Environnement Marin Ressources et risques Naturels. B.P 416. Tanger Principal, 90000, Maroc.

4. Laboratoire Océanographie Spatiale Faculté des Sciences Biologiques, Université Houari Boumediene B.P 32 El Alia 16111 Alger.

\section{Résumé :}

Le choix des littoraux de Béjaïa et Saïdia se justifie par le fait qu'ils sont fortement exposés depuis plusieurs décennies, par les activités de développement notamment urbain et touristique. L'objectif principal de ce travail est d'évaluer le risque d'érosion sur les deux littoraux (algérien et marocain) et surtout à l'impact des changements climatiques pour prévenir les catastrophes, minimiser les risques et gérer les conséquences, une méthodologie de la photo-interprétation a été appliquée pour une base de données s'étalant presque sur une soixantaine d'année.

L'analyse rétrospective de l'évolution morphologique des littoraux a montré : un seul type étant l'érosion fatale pour le littoral de Béjaiia contre trois types principaux d'effets potentiels qui affecteraient la zone marocaine :

- $48 \%$ présentent un risque d'érosion et $22 \%$ en engraissement de la totalité du littoral.

- 1529 m de longueur à Est de Oued Moulouya est la zone la plus touchée par l'érosion côtière.

- Une accélération de l'érosion côtière du secteur Est du littoral.

Tous ces impacts affecteront la biodiversité et les nombreux écosystèmes de ces littoraux, mais aussi les activités socio-économiques de la région.

Ils tiennent à plusieurs facteurs, naturels tels que le changement climatique, et anthropiques dont les aménagements fluviatiles, portuaires et touristiques qui y règnent. Mots clés: Trait de côte, SIG, Érosion côtière, Changements climatiques, Photointerprétation, Béjaïa, Saïdia. 


\section{Thème 2 - Dynamique sédimentaire}

\section{Introduction}

Les zones côtières sont soumises à de nombreuses pressions liées à la présence croissante et envahissante de l'homme. Notre étude porte sur une évaluation comparative de deux portions du littoral nord-africain à savoir : Béjaïa (Algérie) et Saïdia (Maroc).

Le littoral de Saïdia est situé au nord-est du Maroc sur le littoral Méditerranéen, bordé à l'Ouest par Ras Elma et à l'extrémité Est par oued Kiss qui marque la frontière entre le Maroc et l'Algérie, il est linéaire et s'étend sur une longueur de $14 \mathrm{~km}$ environ, de direction ENE-WSW (voir figure 1). Le littoral de Béjaïa est situé au nord-est d'Algérie sur un linéaire de $30 \mathrm{~km}$ du littoral Méditerranéen.

Ainsi, l'objectif principal de ce travail est d'évaluer le risque d'érosion sur les littoraux Algérien et Marocain et surtout à l'impact des changements climatiques pour prévenir les catastrophes, minimiser les risques et gérer les conséquences.

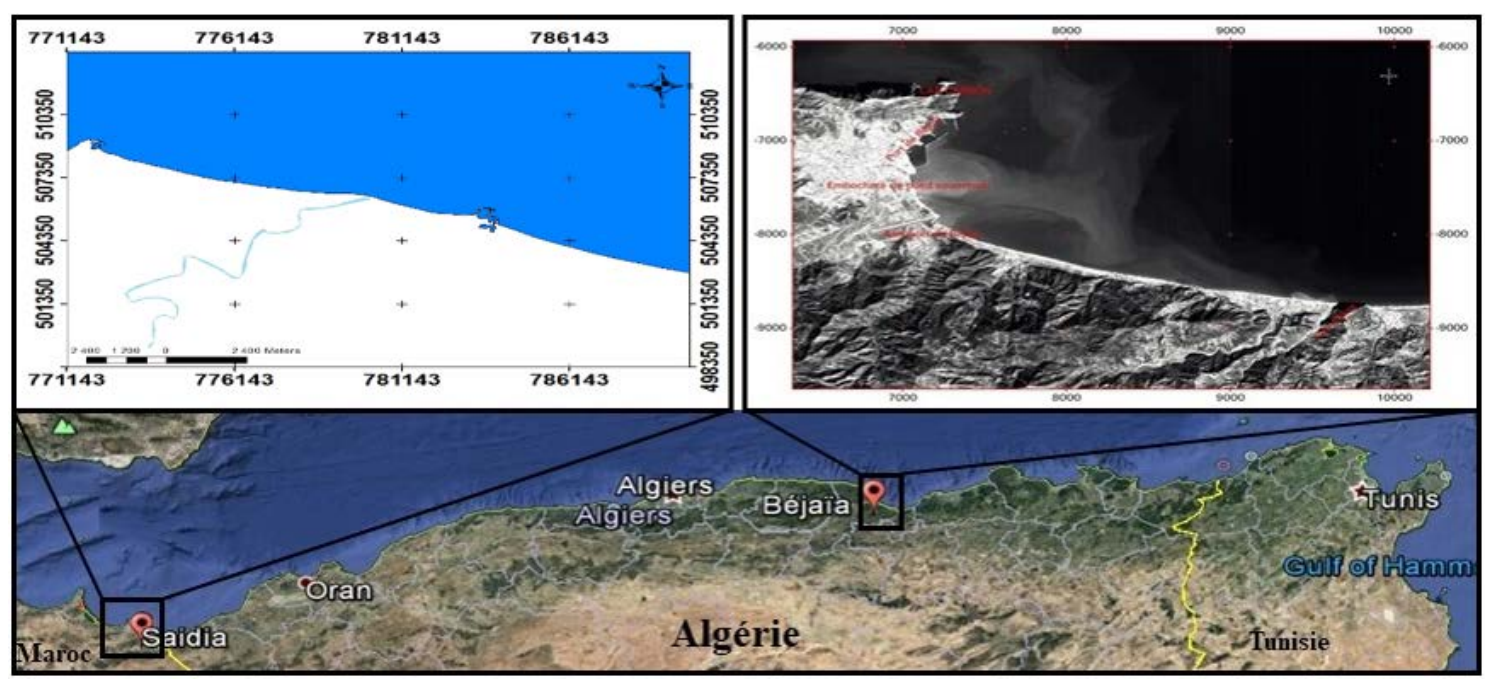

Figure 1. Carte de la localisation des zones d'étude.

\section{Méthodologie}

La méthodologie utilisée s'appuie sur une analyse diachronique des images satellitaires verticales et des cartes topographiques comme document de référence pour servir de base à la correction :

Concernant Saïdia, des cartes de Triffa-Saidia à l'échelle de 1/50 000 et d'Al Hoceima à l'échelle 1/100 000 ont été utilisées à l'aide de l'outil SIG. L'objectif est de réaliser les cartes de risques d'érosion afin d'évaluer les taux de vulnérabilité à l'érosion côtière en se basant aussi sur une étude bibliographique des recherches (MOUZOURI \& IRZI, 2011 ; BOUMEAZA, 1998 ; DAMNATI \& AIT LEMQEDDEM, 2005 ; BOUABDALLAH \& LARUE, 2009).

Les différentes classes d'érosion qui ont été définies sont : forte $(>-3 \mathrm{~m} / \mathrm{an})$, faible $(<-2 \mathrm{~m} / \mathrm{an})$ et stable $(<-0,20 \mathrm{~m} / \mathrm{an})$. 


\section{XVèmes Journées Nationales Génie Côtier - Génie Civil \\ La Rochelle, 29 au 31 mai 2018}

Quant à l'accumulation : forte ( $>+3 \mathrm{~m} / \mathrm{an})$ et stable $(<+0,20 \mathrm{~m} / \mathrm{an})$.

Quant au littoral Béjaoui, nous avons créé quatre (05) couches "layers" sous ArcCatalog, puis, géoréférencé cinq images satellitaires (fournies par l'Institut National de Cartographie et de Télédétection) sous le système de coordonnées horizontales projetées : Nord_Sahara_1959_UTM_Zone_31N.

A l'aide d'ArcMap, nous avons pu digitaliser les traits de côte des cinq images respectivement datant : (1958, 1962, 1985, 2003 et 2016).

Une autre couche, sous le nom "évolution.shp", a été créé dans le but de réunir et superposer les cinq traits de côte issus de la digitalisation.

Les mesures entre les traits ont été faites à l'aide de l'extension Digital Shoreline Analysis System (DSAS) spécialement conçu pour ce genre de travail et compatible avec le logiciel ArcGIS.

Les taux de déplacement annuels ont été calculés sur près de 45 transects ayant une longueur de $300 \mathrm{~m}$ et distants de 200 mètres tout le long du littoral.

Pour mieux cerner le rôle des principaux agents hydrodynamiques dans l'évolution de ces littoraux, une analyse des formes d'érosion et d'accrétion a été effectuée pour déterminer les paramètres morphodynamiques suivant la méthode de Suanez et Provansal (1998) : Le coefficient d'allongement (e) $=\mathrm{D} / \mathrm{d}$ (d, la longueur du petit diamètre et $\mathrm{D}$ la longueur des formes d'érosion ou d'accrétion).

\section{Résultats}

\section{1 Étude de l'évolution morphologique du trait de côte}

\subsubsection{Cas du littoral de Saïdia}

L'étude de l'évolution morphologique du trait de côte au niveau du littoral de Saïdia, montre d'important changement durant la période 1958-2016, avec des zones en érosion $(48 \%)$ et d'autres en engraissement (22\%) sur le reste du littoral (voir figure 2).

La zone a été subdivisée en trois secteurs :

- Le premier couvre la zone de Oued Moulouya, le taux moyen annuel d'érosion côtière a été relativement diminué, il est passé de $-9 ;-10 \mathrm{~m} /$ an pour la période [19582006] à $-5,70 \mathrm{~m} / \mathrm{an}$ pour la période [2009-2016] d'une part, pendant la construction des barrages (barrage Mechrâa Homadi localisé à $60 \mathrm{~km}$ de l'embouchure de Moulouya construit en 1958 et barrage Mohammed V situé à $15 \mathrm{~km}$ en amont du précédent, construit en 1967) la charge sédimentaire accumulé au niveau du littoral, a été fortement érodée.

D'autre part, la crue de 1963 a engendré l'apparition d'une nouvelle embouchure à l'ouest de la précédente par rupture du cordon dunaire, qui survenant dans un contexte sédimentaire défavorable (stock sédimentaire disponible insuffisant). 
Thème 2 - Dynamique sédimentaire
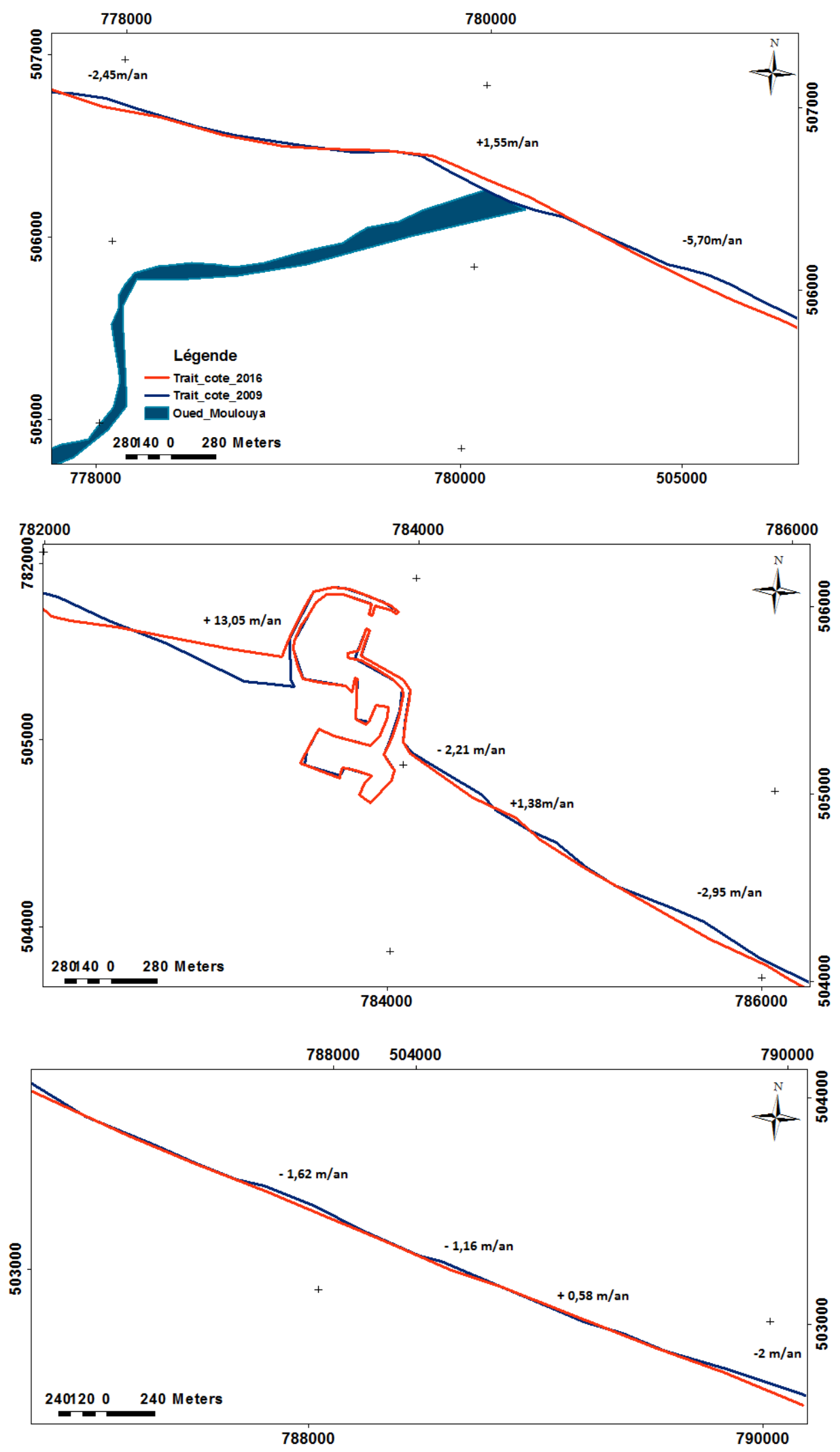

Figure 2. Évolution du Trait de côte de littoral de Saïdia pendant la période 1958-2016. 


\section{XVèmes Journées Nationales Génie Côtier - Génie Civil \\ La Rochelle, 29 au 31 mai 2018}

- Pour le secteur portuaire, on marque une augmentation du taux d'engraissement; En fait, les modifications de la houle engendrées par les jetées ont provoqué une accumulation de sables à ouest $\left[1,85410^{6} \mathrm{~m}^{2}\right.$ pour la période 2009-2016], la jetée du port a été orientée vers l'ouest et a causé de fortes accumulations sableuses en provenance du delta de la Moulouya. Désormais, le port a été agrandi vers l'intérieur des terres entre 2008 et 2009, s'est vu complété d'une nouvelle longue jetée à l'ouest (orientée vers Est) pour protéger l'ancienne entrée.

- Le troisième secteur s'étend entre le port de plaisance et l'extrémité Est du littoral, ce secteur est en érosion alors qu'elle a été relativement stable durant les années 1958 et 2006 (MOUZOURI \& IRZI, 2011 ; BOUABDALLAH \& LARUE, 2009) en raison $\mathrm{du}$ fort hydrodynamisme qui redistribue la fraction fine et transporte avec le bas de la plage. De plus, la construction de la station touristique Saïdia-Méditerrania front de mer occupant une superficie de 713 ha menace également cette zone.

\subsubsection{Cas du littoral de Béjaia}

Les résultats de l'étude de l'évolution morphologique du trait de côte au niveau du littoral de Béjaïa sont représentés par la figure 3.

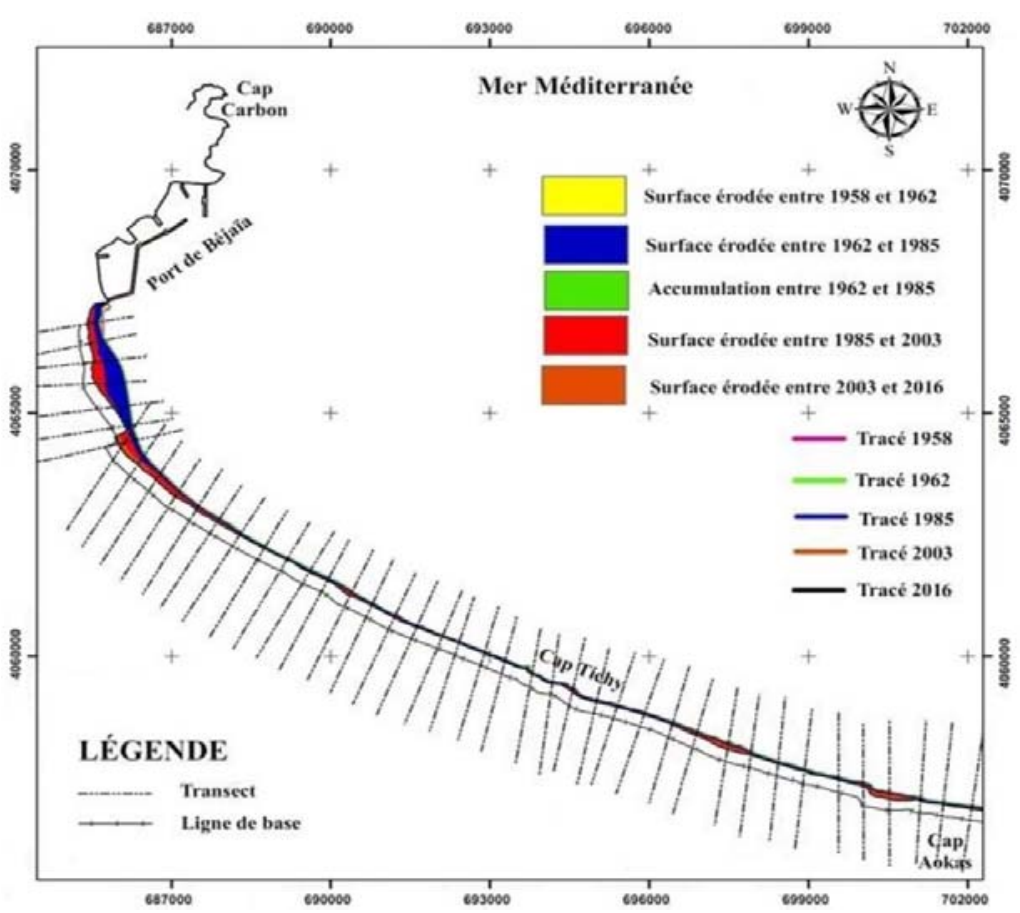

Figure 3. Évolution du Trait de côte de littoral de Béjaïa pendant la période 1958 -2016.

Cette figure représente une analyse diachronique d'une évolution sur une période de 58 ans, ce qui fait apparaitre une forte tendance à l'érosion des plages. À Aokas et une partie de la plage de Tichy, la ligne de rivage a reculé considérablement enregistrant des 


\section{Thème 2 - Dynamique sédimentaire}

taux qui varient entre $-0,52 \mathrm{~m} / \mathrm{an}$ et $-3,49 \mathrm{~m} / \mathrm{an}$. La partie Ouest de la plage de Tichy a connu une alternance de recul et d'accrétion avec une valeur maximale de recul de -1,53 $\mathrm{m} /$ an et un pic d'engraissement atteignant $+1,25 \mathrm{~m} / \mathrm{an}$.

À Boukhlifa et Sidi Ali Lebher, la plage a affiché une récession généralisée sauf une minime portion centrale qui enregistre le taux le plus élevé d'avancée $(+3,73 \mathrm{~m} / \mathrm{an})$. Mais à part cela, les taux nets oscillent entre $-0,02 \mathrm{~m} /$ an et la valeur paroxysmale de $7,19 \mathrm{~m} / \mathrm{an}$.

\section{2. Étude des paramètres morphodynamiques responsable de cette évolution}

\subsubsection{Cas du littoral de Saïdia}

Les résultats de cette analyse montrent que le processus Morphodynamique contrôlant la dynamique sédimentaire est caractérisé par la dominance des inondations qui pourraient être le résultat d'une fréquence accrue de tempêtes et/ou des faibles apports fluviatiles qui se manifeste par une forte érosion du secteur Est d'Oued Moulouya.

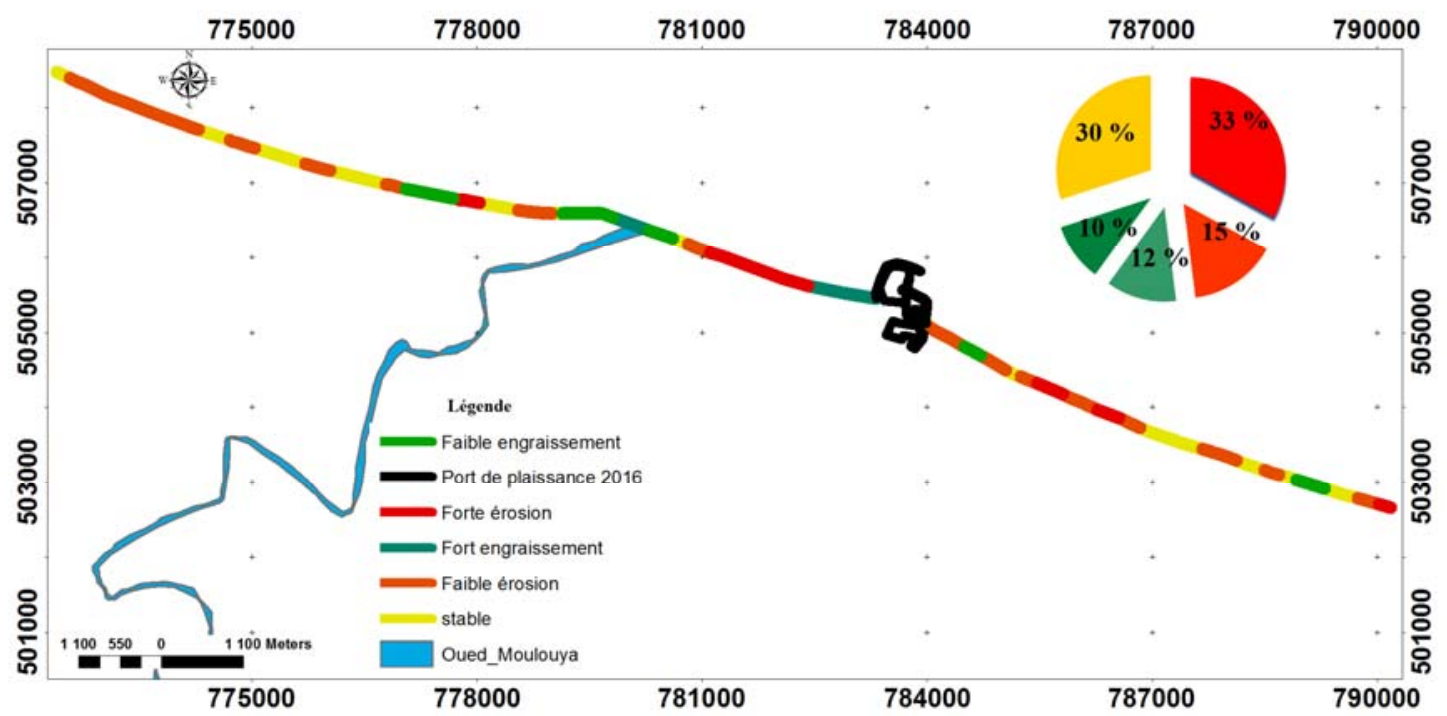

Figure 4. Carte des risques d'érosion du littoral de Saïdia pendant la période 1958-2016.

Cette instabilité tient à plusieurs paramètres naturels (variabilité climatique, distribution temporelle des marées, fréquence des tempêtes.) ou anthropiques (prélèvements de sédiments, piétinement des massifs dunaires, blocage du transit hydrosédimentaire par des ouvrages portuaires ou de défense contre la mer...).

\subsubsection{Cas du littoral de Béjaïa}

La présente étude a révélé que près de $7 \mathrm{~km}$ de côte ont un faible risque, enregistrant des taux d'accroissement oscillant entre $+0,75 \mathrm{~m} / \mathrm{an}$ et $+1,89 \mathrm{~m} /$ an le long des tronçons côtiers ouest et central du Souk El Tenine. $14 \mathrm{~km}$ de côte ont un niveau de risque 


\section{XVèmes Journées Nationales Génie Côtier - Génie Civil \\ La Rochelle, 29 au 31 mai 2018}

modéré, alternant entre érosion et accrétion, avec des taux variant entre $-0,75 \mathrm{~m} / \mathrm{an}$ et +1,89 m/an, le long des littoraux de Tichy, Aokas Est et Souk El Tenine. Environ 19 km de littoral sont d'un risque élevé à très élevé, enregistrant des taux d'évolution oscillant entre $-0,75 \mathrm{~m} / \mathrm{an}$ et $-2,25 \mathrm{~m} / \mathrm{an}$ le long des tronçons côtiers de Boukhlifa, Sidi Ali Lebher, Melbou et Aokas ouest.

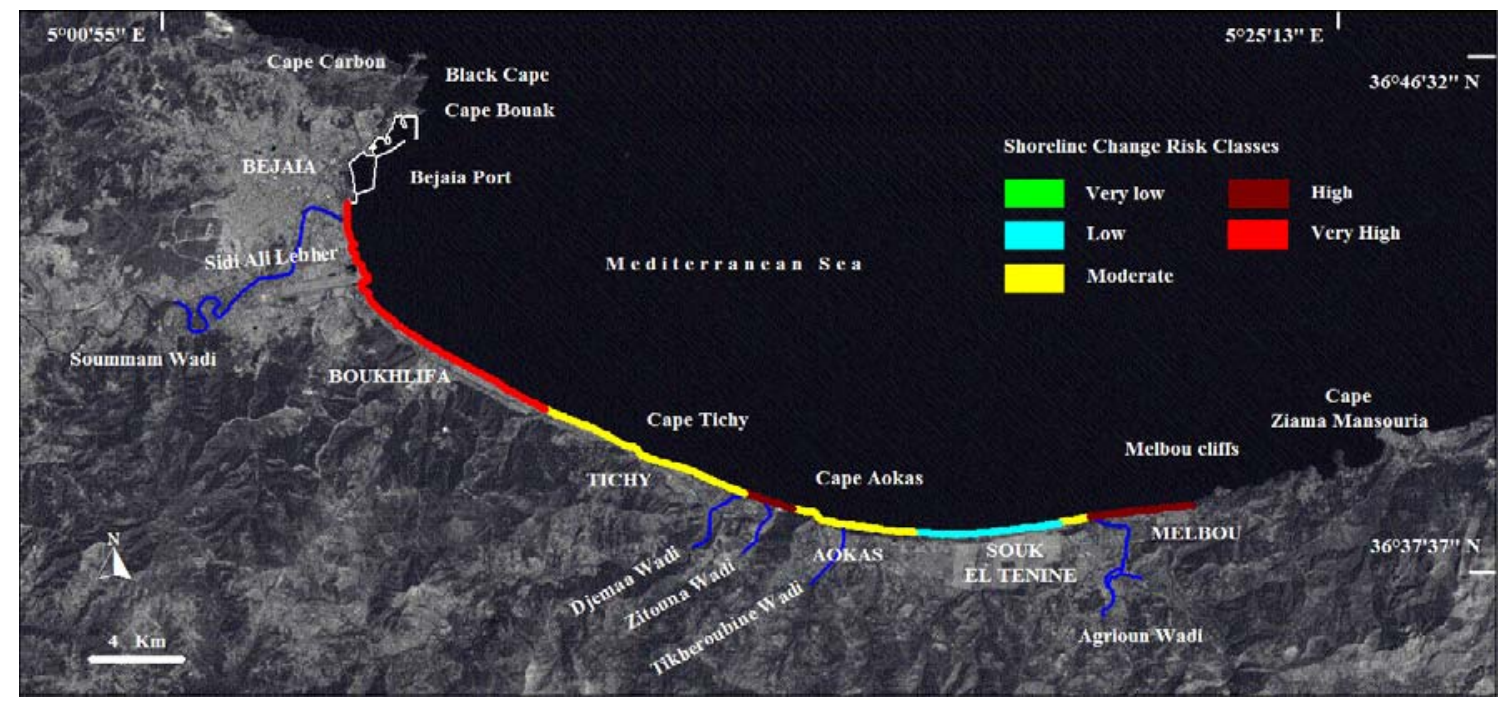

Figure5. Carte des risques d'érosion du littoral de Béjaïa pendant la période 19582016 (DJOUDER \& BOUTIBA, 2017).

\section{Conclusions}

Cette étude nous a montré un retrait de la ligne de rivage. Ce dernier pourrait être causé par une variabilité climatique traduite par des périodes de crue et vu de l'importante dérive sédimentaire vers l'Est, ceci affectant principalement le secteur Est de Moulouya; et anthropique, suite à la construction d'une station touristique nommée: SaïdiaMéditerrania.

En effet, le suivi de l'évolution du littoral Est Béjaoui a fait ressortir que la récession des plages est un phénomène ancien, il met en évidence une action néfaste et successive de faits probablement : météorologiques, hydrodynamiques et humains. Cette érosion ne s'est pas atténuée au fil du temps, bien au contraire, elle a continué à sévir enfonçant encore plus le littoral dans une déroute automatiquement incontrôlable.

Ceci mérite de réelles réflexions sur la protection et la gestion de ce littoral surtout que les perspectives des changements climatiques (d'ici 2100, la température moyenne de l'air à la surface du globe devrait augmenter de $1,1^{\circ} \mathrm{C}$ à $6,4^{\circ} \mathrm{C}$ par rapport à 1990 : GIEC, 2007) et de l'élévation du niveau de la mer (Les modèles prévoient une élévation supplémentaire de ce niveau de 9 à $88 \mathrm{~cm}$ d'ici l'an 2100 : GIEC, 2007) affecteraient fortement ces zones. 


\section{Thème 2 - Dynamique sédimentaire}

Les résultats obtenus appellent à une réflexion objective sur la gestion actuelle de cette côte et de l'ensemble des littoraux Algérien et Marocain. A cette fin, des études similaires devraient être menées à travers l'ensemble du littoral Méditerranéen afin de caractériser l'érosion globale de ses côtes.

\section{Remerciements}

Nos remerciements s'adressent à l'ensemble de notre équipe de recherche, l'initiative "science-maghrébine" ainsi qu'à tous ceux qui ont organisé les XV $\mathrm{X}^{\mathrm{eme}}$ Journées Nationales Génie Côtier - Génie Civil et en particulier Professeur Daniel LEVACHER.

\section{Références bibliographiques}

BOUABDALLAH M., LARUE J.P. (2009). Evolution du littoral de la baie de Saïdia : Dynamique naturelle et impact des aménagements (Maroc Oriental) Revue PhysioGéo, Vol. 3, pp 113-130. https://doi.org/10.4000/physio-geo.878

BOUMEAZA T. (1998). Morphologie et évolution du delta et du littoral de la basse Moulouya, Maroc nord-oriental : apport des images aéroportées et satellitales. Géoobservateur, Vol. 8, pp 65-77.

DAMNATI B., AIT LEMQEDDEM KH. (2015). Impact des changements climatiques sur le littoral Méditerranéen : exemple du littoral de Saïdia et de Tanger. Recueil. 22ème colloque international des bassins sédimentaires. Volume des résumés, pp 215-218.

DJOUDER F., BOUTIBA M. (2017). Vulnerability assessment of coastal areas to sea level rise from the physical and socioeconomic parameters: case of the Gulf Coast of Bejaia, Algeria. Arabian J. Geosciences, Vol. 10: 299. https://doi.org/10.1007/s12517-017$\underline{3062-5}$

MOUZOURI M., IRZI Z. (2011). Évolution et morpho-dynamique de la plaine côtière de Saïdia : littoral méditerranéen du Nord-Est du Maroc durant la période 1958-2006. Bulletin de 1'Institut Scientifique, Rabat, section Sciences de la Terre, ${ }^{\circ} 33$, pp 65-76. 